as the oral contraceptive pill (OCP) removed the fear of pregnancy for many women. While that may contribute to an increase in STI, as the OCP did, we should use this as an opportunity to argue for renewed investment in STI control programmes and research.

Disclosure No significant relationships.

\section{SO3.2 THE ROLE OF ORAL-ANAL TRANSMISSION IN PERSISTENCE OF CHLAMYDIAL INFECTION}

Henry De Vries*. Amsterdam University Medical Centres, Dermatology, Amsterdam, Netherlands

\subsection{6/sextrans-2019-sti.25}

In virtually every mammal, chlamydiae strains persist in the gastro-intestinal tract for long periods of time in the absence of apparent inflammation and pathology. Although anorectal Chlamydia trachomatis (CT) infections are diagnosed frequently in women and men who have sex with men, their detection often remains unexplained as anal sex is not always reported. Oropharyngeal infections and the gastro-intestinal tract may contribute to the explanation. Both symptomatic and asymptomatic chlamydial pharyngitis has been described. Consequentially, the pathogen may pass through the gastrointestinal tract to the large intestine and rectum, inducing chlamydia proctitis, and ongoing transmission. Oro-anal transmission might also explain the asymmetric distribution of diagnosed anorectal and urogenital lymphogranuloma venereum infections among men who have sex with men as found in the current epidemic. Recently the use of saliva as lubricant for anal sex has been proposed as alternative (oro-anal) route for gonorrhoea transmission in MSM. It can be deduced that this mode of transmission further contributes to the exceeding numbers of anorectal LGV infections as opposed to genital infections.

Disclosure No significant relationships.

\section{S03.3 SEXUAL TRANSMISSION OF N. MENINGITIDIS IN MSM AND IMPLICATIONS FOR PUBLIC HEALTH}

Anna McNulty*. Sydney Sexual Health Centre, Sydney, Australia

\subsection{6/sextrans-2019-sti.26}

Over the last 5 years we have seen outbreaks of N. meningitidis urethritis and invasive meningococcal disease (IMD) in gay and bisexual men in various centres around the world with speculation as to what has driven these outbreaks. As a result there has been a significant focus on the genomics of the isolates associated with the recent outbreaks and in particular horizontal gene transfer from $N$. gonorrhoeae and the impact on the virulence of $N$. meningitidis Vaccination against meningococcus has impacted the epidemiology of this infection broadly however the impact in MSM is less clear. There is relatively little recent data about the prevalence of asymptomatic carriage in MSM particularly in the era of PrEP. Commonly, STI management guidelines do not include sexually transmitted $N$. meningitidis and there are varying laboratory practices with regard to notification of isolates and public health guidance is variable as well. In this presentation the epidemiology of N. meningitidis in MSM around the world will be explored. The potential drivers of these outbreaks will be examined and in particular the genomic investigations into clonal complex 11. The public health responses to date and the implications for public health as well as STI clinicians will be examined.

Disclosure No significant relationships.

\section{SO3.4 HEPATITIS C IN HIV-NEGATIVE MSM - A GROWING CONCERN?}

Axel Schmidt*. London School of Hygiene and Tropical Medicine/SIGMA Research, London, UK

\subsection{6/sextrans-2019-sti.27}

In post-industrialised countries, since the turn of the millennium we have witnessed outbreaks of hepatitis C among HIVdiagnosed gay men, and cohort studies of HIV-diagnosed men-who-have-sex-with-men (MSM) have shown high incidence of HCV infection, or re-infection after clearance. Clinicians and health promotion specialists have repeatedly expressed concerns that the HCV epidemic will jump to HIVnegative MSM. For the last two decades there has been a widespread perception that HCV will inevitably affect the whole population of MSM. For many years, studies of HIVnegative MSM have provided no strong evidence for an HCV sero-prevalence higher than among other adult men, and no outbreaks had been observed among HIV-negative MSM. It has hence been conceptualised that HIV infection itself might be a major risk factor for HCV acquisition, due to alterations of cellular immunity, but the risk attributable to immune dysfunction is still unclear. Some have argued that sero-adaptive sexual networks might better explain the observed differences between HIV-diagnosed and other MSM. As for behavioural factors, while the role of condomless anal sex for HCV transmission is controversial, there is consensus that mucosal disruption and sexual exposure to blood are major risk factors. With the recent advent of oral HIV pre-exposure prophylaxis, shifts in sexual networks have been observed as well as rising $\mathrm{HCV}$ incidence in PrEP-using HIV-negative MSM. This reignites previous fears about the imminence of an HCV epidemic among HIV-negative MSM. While intravenous drug use with shared equipment has been known for decades to be a leading risk factor for HCV transmission -regardless of HIV infection or sexual orientation- its impact on HCV transmission in MSM is understudied. This talk aims at (1) summarising the available literature on HCV transmission, focussing on HIVnegative MSM, and (2) providing a conceptual framework of sexual and sex-associated HCV transmission in gay men.

Disclosure No significant relationships. 